\title{
SOME REMARKS ABOUT MACKEY CONVERGENCE
}

\author{
JÓZEF BURZYK \\ Institute of Mathematics \\ Polish Academy of Science \\ Wieczorka 8 \\ Katowice, 40-013, Poland
}

THOMAS E. GILSDORF

Department of Mathematics

University of North Dakota

Grand Forks, ND 58202-8376, USA

(Received September 16, 1993 and in revised form March 5, 1994)

\begin{abstract}
In this paper, we examine Mackey convergence with respect to $K$ convergence and bornological (Hausdorff locally convex) spaces. In particular, we prove that: Mackey convergence and local completeness imply property $K$; there are spaces having $K$ - convergent sequences that are not Mackey convergent; there exists a space satisfying the Mackey convergence condition, is barrelled, but is not bornological; and if a space satisfies the Mackey convergence condition and every sequentially continuous seminorm is continuous, then the space is bornological.
\end{abstract}

KEY WORDS AND PHRASES. Mackey convergence, property $K$, barrelled space, bornological space.

1991 MATHEMATICS SUBJECT CLASSIFICATION CODE. Primary 46A17; Secondary 46A08.

\section{INTRODUCTION.}

This note contains a somewhat miscellaneous collection of results that do or do not hold for a space ("space" here means: Hausdorff locally convex space) that satisfies the Mackey convergence condition; specifically, we consider the following properties of a space $(X, \tau)$ :

(a) $(X, \tau)$ satisfies the Mackey convergence condition [1;5.1.29, page 158]: For every null sequence $(x(n))$ there is there is a closed, bounded absolutely convex set (i.e., a disk ) $B$, such that $x(n) \rightarrow 0$ in $X_{B}$, where $X_{B}$ is the linear span of $B$ equipped with the topology of the gauge of $B$. Equivalently, $[1 ; 5.1 .3$, page 151] for every null sequence $(x(n))$ there is a sequence $(a(n))$ of positive numbers such that $\mathrm{a}(\mathrm{n}) \rightarrow \infty$ as $n \rightarrow \infty$, and $\mathrm{a}(\mathrm{n}) \mathrm{X}(\mathrm{n}) \rightarrow 0$ in $X$. 
(b) $(X, \tau)$ satisfies the Katowice property (also property $K$ or is a $\underline{K}$ - $\underline{\text { space) }}$ [1;1.2.15, page 20], [2; page 19]: For every null sequence $(x(n))$ there is a subsequence $(y(n))$ of $(x(n))$ such that $\sum_{n=1}^{\infty} y(n)$ converges in $X$.

(c) $(X, \tau)$ is bornological.

Spaces that satisfy (a) have been studied recently with regard to topics such as inductive limits. For example, the reader may peruse [3] and the references therein. Property (b) was originally defined by Mazur and Orlicz (see [1; 1.4, page 30]) and was used in [4] to find some sequential conditions under which a space is bornological.

We first show that Mackey convergence and local completeness imply property $K$, where we recall that a space $(X, \tau)$ is locally complete $[1 ; 5.1 .6$, page 152] if for each closed bounded disk $B$, the space $X_{B}$ is a Banach space. However, we also prove that there are spaces having $K$-convergent sequences that are not Mackey convergent and that a weak Mackey convergent sequence is a Mackey convergent sequence for the original topology. Hence, a space that satisfies property (a) with respect to its weak topology also satisfies Shur's property- every weakly convergent sequence is convergent for the original topology.

Antosik and the first author ask in [4] whether a barrelled space for which every sequentially continuous seminorm is continuous must be bornological. Bonet [5] proves the answer is "no". We prove here that if the barrelled assumption is replaced by (a), then the answer is "yes". This slightly improves the last result of [4].

Finally, we exhibit a space that satisfies (a), is barrelled, but is not bornological. This example is related to an old problem of Grothendieck (see [6] and [7]): Is there a quasibarrelled (DF) -space satisfying (a) but not bornological?

\section{MAIN RESULTS.}

THEOREM 1. Let $(\mathrm{X}, \tau)$ be a space.

(i) If $(\mathrm{X}, \tau)$ is locally complete and satisfies the Mackey convergence condition, then it satisfies property $K$.

(ii) Any weak Mackey convergent sequence is Mackey convergent with respect to the original topology.

(iii) If $(\mathrm{X}, \tau)$ satisfies the Mackey convergence condition with respect to its weak topology, then every weakly convergent sequence is originally convergent in $X$. 
PROOF: (i). Suppose $(x(n))$ is any null sequence in $(X, \tau)$. Local completeness and the Mackey convergence condition together imply that $(x(n))$ is a null sequence in $X_{B}$ for some closed bounded disk $B$, and $X_{B}$ is a Banach space. As a Banach space satisfies property $K,(x(n))$ has a subsequence that is series convergent in $X_{B}$ to some $x \in X_{B}$. Because $B$ is closed and the identity map from $X_{B}$ into $X$ is continuous, this subsequence is series convergent in $X$.

(ii). Now suppose $(x(n))$ is a weak Mackey convergent sequence in $X$. Then there is a sequence $(a(n))$ of positive numbers tending to infinity, and such that $a(n) x(n) \rightarrow 0$ weakly. The set $\{a(n) x(n): n \in N\}$ is even $\tau$-bounded in $X$. Therefore, it suffices to find a sequence $(\lambda(n))$ of positive numbers such that $\lambda(n) \rightarrow 0$ but $a(n) \lambda(n) \uparrow \infty$.

(iii). Obvious consequence of (ii).

The authors do not know if property $K$ implies the Mackey convergence condition in general, but the next example shows that one can have a sequence that satisfies property $K$ but is not Mackey convergent.

EKAMPLE 1. Let $X=l^{\infty}$, equipped with the following seminorms: For each $x=(x(n)) \in X$, define:

(a) For each $k \in N, p_{k}(x)=|x(k)|$.

(b) For each $y \in I^{1}, p_{y}(x)=\sum_{k=1}^{\infty} y(k) x(k)$.

We let $\tau$ be the locally convex topology generated by these seminorms. Note that by our definition of $\tau$, a sequence $(x(n))$ is $\tau$-convergent to $x \in X$ if and only if:

(i) $x(n) \rightarrow x$ coordinatewise; namely, $x(n, k) \rightarrow x(k)$, as $n \rightarrow \infty$, and

(ii) There is an $M>0$ such that for every $k, n \in \mathrm{N},|x(n, k)| \leq M$.

Define $(z(n)) \subset X$ by $z(n)=e(n)$, where $e(n)=(0,0, \ldots, 0,1,0, \ldots) \quad(1$ in the $n$th place). Clearly, $z(n) \rightarrow 0$ and is even summable, hence $(z(n))$ is a $K$ sequence. However, because of (i) and (ii), there cannot be a sequence $(a(n))$ of positive numbers tending to infinity such that $a(n) z(n) \rightarrow 0$.

We note here that the above space does not satisfy property $K$; consider the sequence $z(n)=(0,0, \ldots, 0,1,1, \ldots) \quad(0$ in the first $n-1$ places, then 1 's $) .(z(n))$ is a null sequence in $X$, but has no summable subsequence.

THEOREM 2. If $(X, \tau)$ satisfies the Mackey convergence condition and every sequentially continuous seminorm on $X$ is continuous, then $(X, \tau)$ is bornological.

PROOF. Assume that $p$ is a seminorm on $X$ such that if $x(n) \rightarrow 0$ then 
$(p(x(n)))$ is bounded. Then there is a sequence of positive scalars $\alpha(n) \rightarrow \infty$ such that $\alpha(n) \times(n) \rightarrow 0$, which in turn means that for some $M>0, p(\alpha(n) x(n)) \leq M$ for each positive integer $n$. Hence, $p(x(n)) \rightarrow 0$. This shows that $p$ is sequentially continuous, and by assumption, $p$ is continuous. It follow's that $(X, \tau)$ is bornological.

We now present an example of a space that satisfies the Mackey convergence condition, is barrelled, but is not bornological. However, we will also show that the space does not have a fundamental sequence of bounded sets so is not a solution to the Grothendieck problem.

EKAMPLE 2. Let $X$ be the set of all real-valued functions on $\mathrm{R}$ that are constant except on a countable set. Equip $X$ with the locally convex topology $\tau$ generated by the family of seminorms $P=\left\{p_{t}: t \in \mathbf{R}\right\}$, where

$$
p_{t}(x)=|x(t)|, \quad x \in X
$$

Claim $1:(X, \tau)$ satisfies the Mackey convergence condition. To see this, suppose $(x(n))$ is any null sequence in $(X, \tau)$. For each $n \in \mathrm{N}$, let $T_{n}$ denote the set on which $(x(n))$ is not constant. We count $T_{n}$ by $T_{n}=\{t(1, n), t(2, n), \ldots\}$. Denoting by 1 the characteristic function, we may represent $x(n) \in X$ by

$$
x(n, t)=c(n)+\sum_{k=1}^{\infty} \lambda(n, k) 1\{(n, k)\}
$$

where $c(n)$ and $\lambda(n, k)$ are constants, for $n, k \in N$. It is clear from this that $(c(n))$ is a null sequence of real numbers. Next, denote by $T$ the countable set $\cup\left\{T_{n}: n \in N\right\}$, and label it by $T=\{t(1), t(2), \ldots\}$. Because $x(n, t(k)) \rightarrow 0$ as $n \rightarrow \infty$, there is a sequence $(a(n)) \uparrow \infty$ such that both $a(n) x(n, t(k)) \rightarrow 0$ and $a(n) c(n) \rightarrow$ 0 , as $n \rightarrow \infty$. Thus, $(x(n))$ is a Mackey convergent sequence.

Claim $2:(\mathrm{X}, \tau)$ is barrelled. If $f \in X^{\prime} \quad\left(X^{\prime}\right.$ denotes the continuous dual of $\left.X\right)$, then $f$ is of the form

$$
f(x)=\alpha(1) x(t(1))+\ldots+\alpha(k) x(t(k))
$$

for $\{t(1), \ldots, t(k)\} \subset R$, and some nonzero scalars $\alpha(1), \ldots, \alpha(k)$. To prove that $X$ is barrelled, it is enough to show equicontinuity for sequences of 
pointwise bounded members of $X^{\prime}$. With this in mind, let $(f(n))=(f(n, x))$ be any sequence such that for every $x \in X$ and every $n \in N,|f(n, x)|$ is bounded. We will find a $C>0$ and a $p \in P$ such that $|f(n, x)| \leq C p(x), n \in N, x \in X$.

For each $n \in N$, let $S_{n}=\left\{t(1, n), t(2, n), \ldots t\left(k_{n} \cdot n\right)\right\}$, for which

$$
f(n, x)=\sum_{i=1}^{h_{4}} \alpha(i, n) x\left(t\left(k_{i}, n\right)\right)
$$

$\alpha(i, n) \neq 0$ constants. Put

$$
S=\cup\left\{S_{n}: n \in N\right\}
$$

$S$ is finite; for if not, then there would exist for each $n \in \mathbf{N}$,

$t(n) \in S_{\beta_{n}} \backslash\left[\cup\left\{S_{k}: k=1, \ldots, \beta_{n-1}\right\}\right]$. For brevity, relable $\beta_{n}$ by $n$, and then denote by $y$ an element of $X$ that takes the values given by $y\left(t\left(k_{n}, n\right)\right)=$ $\left.l \alpha\left(k_{n}, n\right)\right\}^{-1} n$. Then $f(n, y)=n k_{n} \rightarrow \infty$ as $n \rightarrow \infty$, and this is bad because then $(f(n))$ is not pointwise bounded at $y$.

Hence, we are allowed to write $S=\{t(1), t(2), \ldots t(K)\}$ for some $K \in \mathrm{N}$, and

$$
f(n, x)=\sum_{i=1}^{K} \alpha(i, n) x\left(t_{i}\right) .
$$

Put $\alpha(i)=\max \{|\alpha(i, n)|: n \in \mathrm{N}\}$, for $i=1, \ldots, K$. Each $\alpha(i)<\infty$ because of the pointwise boundedness of the sequence $(f(n))$. It follows that with $\mathrm{C}=\max \{\alpha(i):, i=1, \ldots, K\}$, we have

$$
\begin{aligned}
|f(n, x)| & \leq \sum_{i=1}^{K} \alpha(i)\left|x\left(t_{\dot{i}}\right)\right| \\
& \leq C p(x)
\end{aligned}
$$

where $p$ is the seminorm corresponding to the set $S$. This means that $(f(n))$ is equicontinuous.

Claim $3:(X, \tau)$ is not bornological. To see this, consider the linear functional $f$ on $X$ given by $f(x) \equiv c$, where $x(t)=c$, except on a countable set. $f$ maps bounded sets in $X$ to bounded sets in $\mathrm{R}$, but is not continuous; hence by [1; 6.1 .18 , page 168 ], $X$ cannot be bornological. 
Claim $4:(X, \tau)$ does not have a fundamental sequence of bounded sets (f.s.b.). Io prove this, suppose for a contradiction that $(X, \tau)$ has a f.s.b., $\left(B_{n}\right)$. Then in particular, for each $n=1,2, \ldots$, we can find $a_{n}>0$ such that for each $x \in B_{n}$, $|x(n)| \leq a_{n}$. Let us define $A \subset X$ by $A=\left\{y_{m}: m \in N\right\}$, where we define each $y_{m}$ as follows: put $y_{m}(t)=0$ if $t \neq m$, and $y_{m}^{\prime}(m)=1+a_{m}$. From this we see that for any $t \in \mathrm{R}$, we have that if $t=m_{0}$ for some $m_{0} \in \mathrm{N}$, then $\sup \left\{\left|y_{m}(t)\right|: m \in N\right\}$ is $1+a_{m_{0}} ;$ otherwise $\sup \left\{\left|y_{m}(t)\right|: m \in N\right\}=0$. Therefore, $A$ is $\tau$-bounded. On the other hand, it is easy to see that $A$ is not contained in any $B_{n}$.

ACKNOWLEDGEMENT. Research for the second named author was supported by the University of North Dakota. The authors would also like to thank Professor P. Antosik and the referee for several useful comments concerning this paper.

\section{REFERENCES}

[1] PÉREZ-CARRERAS, P., BONET, J. Barrelled Locally Convex Spaces, North Holland Math. Studies no. 131, 1987.

[2] SWARTZ, C. An Introduction to Functional Analysis, Dekker, 1992.

[3] BIERSTEDT, K. D. An introduction to locally convex inductive limits, in Functional Analysis and its Applications, World Sci. Pub., 1987.

[4] ANTOSIK, P., BURZYK, J. Sequential conditions for barrelledness and bornology, Bull. Pol. Acad. Sci., 35 no. 7-8, (1987), 457-459.

[5] BONET, J. A question of Antosik and Burzyk on non-bornological barrelled spaces, Bull. Pol. Acad. Sci., 39, no. 3-4, (1991), 175-176.

[6] PÉREZ-CARRERAS, P. Una nota sobre espacios DF, Rev. Real Acad. Ci. Madrid, 75, (1981), 739-741.

[7] VALDIVIA, M. A class of quasibarrelled (DF) spaces which are not bornological, Math. Z., 136, (1974), 249-251. 


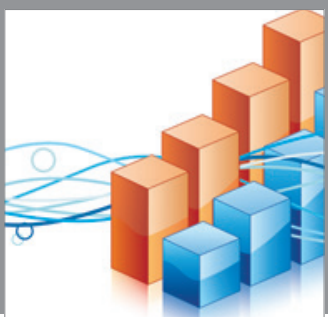

Advances in

Operations Research

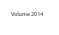

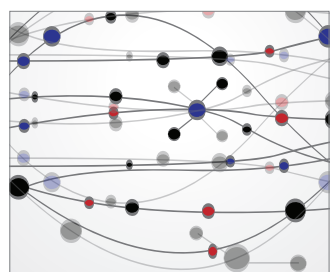

\section{The Scientific} World Journal
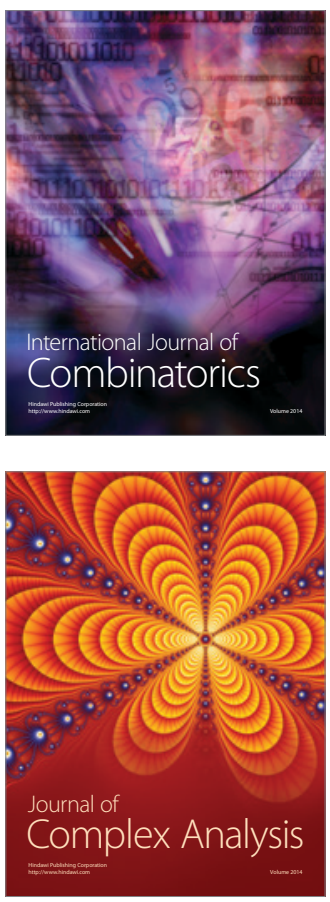

International Journal of

Mathematics and

Mathematical

Sciences
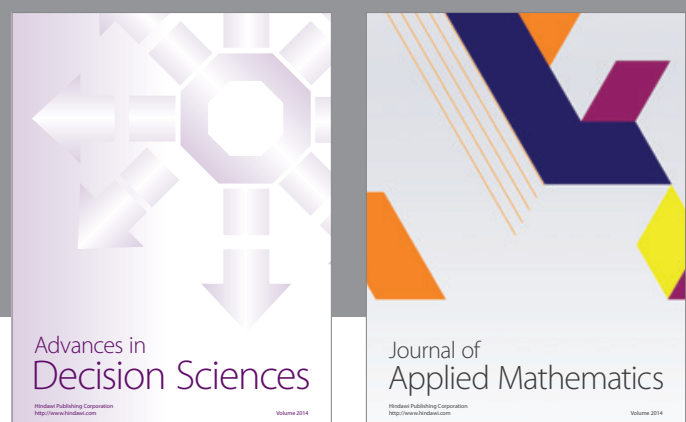

Journal of

Applied Mathematics
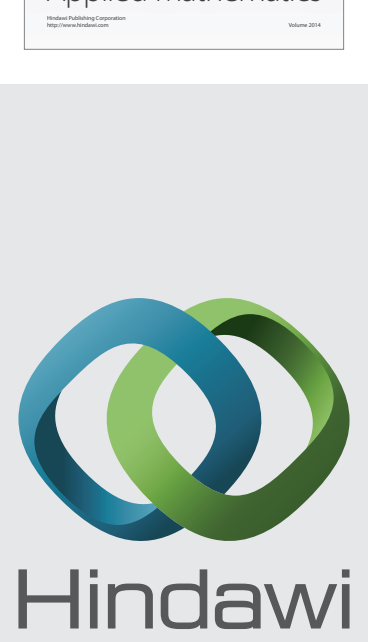

Submit your manuscripts at http://www.hindawi.com
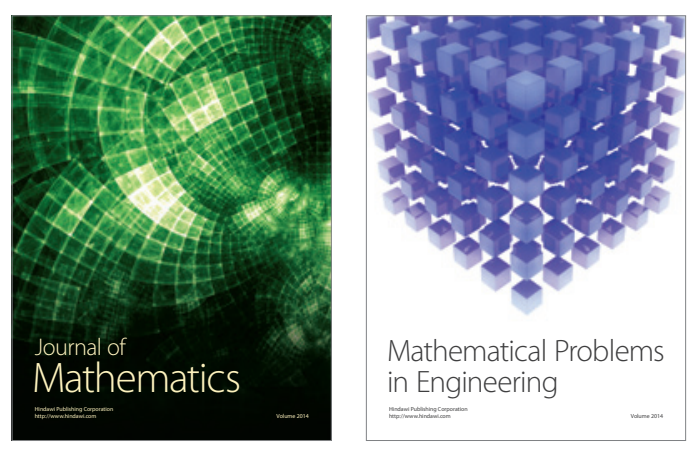

Mathematical Problems in Engineering
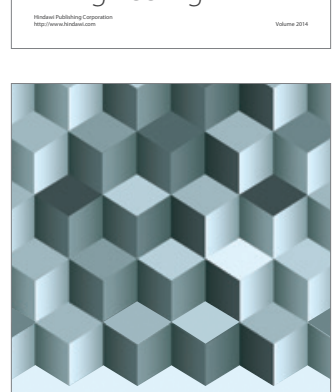

Journal of

Function Spaces
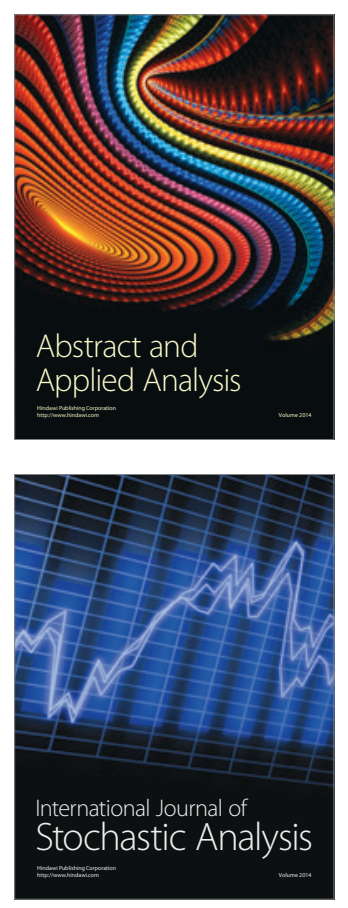

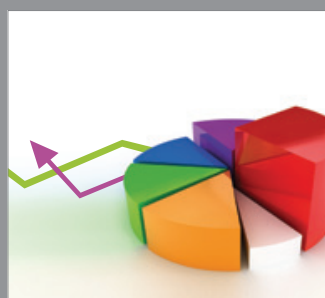

ournal of

Probability and Statistics

Promensencen
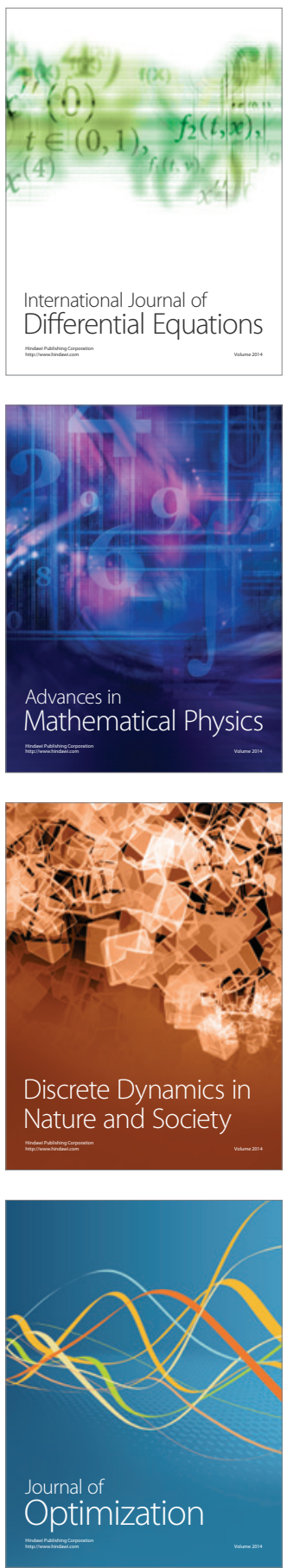